\title{
A Rare Morphological Study Concerning the Longest Bone of the Human Anatomy in the Population of the Northern Greece
}

\author{
George Noussios $^{\mathrm{a}}$, Konstantinos Theologou ${ }^{\mathrm{b}}$, Pantelis Chouridis ${ }^{\mathrm{c}}$, George Karavasilis ${ }^{\mathrm{d}}$, \\ Grigorios Alafostergios ${ }^{\mathrm{e}}$, Anastasios Katsourakise, $\mathrm{f}$
}

\begin{abstract}
Background: The femur is one of the most researched bones in the human anatomy and forensic medicine. As the longest bone in the human body, it is well preserved in skeletal remains. The sex estimation of human remains is one of the most important research steps for physical and forensic anthropologists. However, osteometric standards built on unburned human remains and contemporary cremated series are often inadequate for the analysis, frequently resulting in a significant number of misclassifications.
\end{abstract}

Methods: In our study, we present the anthropometric data from 500 skeletons in Northern Greece, including 232 males and 198 females, as well as 430 of known age. The diameters of the femur were measured as well as the indices of robustness. For the statistical interpretation of the results, we have used the discriminant analysis.

Results: From the interpretation of the data, we concluded that all the mean values, diameters and indices of the males were greater compared with those of the females. Also, we concluded that the probability of error is quite high in all cases except the vertical diameter of femur's head, which has an acceptable percentage of error of $14.39 \%$ and can be used as a safe criterion for sex identification.

Conclusion: With the aid of statistics, we came to the conclusion that the vertical diameter of the femur's head is a safe variable for sex estimation in skeletal remains.

Keywords: Femur; Anatomy; Anthropometric data; Sex identification

Manuscript submitted September 20, 2019, accepted October 9, 2019

a'Laboratory of Anatomy, School of Physical Education and Sports Sciences at Serres, "Aristotle" University, Thessaloniki, Greece

bOrthopedic Surgeon, Thessaloniki, Greece

'ENT Police Medical Centre, Thessaloniki, Greece

${ }^{\mathrm{d}}$ Department of Business Administration, Technological Educational Institute of Central Macedonia, Serres, Greece

eDepartment of Surgery, Agios Dimitrios General Hospital, Thessaloniki, Greece

${ }^{f}$ Corresponding Author: Anastasios Katsourakis, Department of Surgery, Agios Dimitrios General Hospital of Thessaloniki, Elenis Zografou 2, GR-54634, Thessaloniki, Greece. Email: tasoskatsourakis@hotmail.com

doi: https://doi.org/10.14740/jocmr3986

\section{Introduction}

The femur is one of the most well-described bones of the human skeleton in fields ranging from clinical anatomy to forensic medicine. Because it is the longest and strongest bone in the human body, and thus, one of the most well-preserved in skeletal remains, it makes the greatest contribution to archaeology.

In this study, we present anthropometric data on the femur from a population in northern Greece. A common acknowledgement is that there are variations among different ethnicities in the skeletal characteristics, such as bone form and dimensions. We provide data for distinguishing the femur according to sex and race.

\section{Materials and Methods}

The current study took place in the ossuary of the town of Thessaloniki, where skeletal remains have been kept since 1976. In total, 500 skeletons of known sex were measured (232 males and 198 females), of which, 430 were of known age. The vast majority of the remains belonged to people over 40 years old. We have included into the study only the skeleton remains of humans that were above the age of 18 . All the deceased people were of the Caucasian race, living in the town of Thessaloniki in the Mediterranean area. Bones that were brittle or partially destroyed were excluded from the research.

The following diameters of the bones were measured (excluding the far smaller and more brittle fibula): the maximal length of the femur (MLF), natural length of the femur (NLF), distance between the greater trochanter and head (GTH), distance between the greater and lesser trochanters (GLT), vertical diameter of the head $(\mathrm{VDH})$, perimeter in the middle of the femur body (PMF) and distance of lateral and medial (DLM) between the outermost extremities of the two condyles of the femur. We also calculated the indices of robustness using the following mathematical formulas: index of robustness of the femur $1(\operatorname{IRF} 1)=\mathrm{PMF} / \mathrm{NLF} \times 100$ and IRF $2=\mathrm{PMF} / \mathrm{MLF}$ $\times 100$.

For the statistical interpretation of the results, we used the discriminant analysis of only one dimension, the length of the bone, because the dependent variable is categorical and the 
Table 1. The Mean Values and the Standard Deviation of the Measurements

\begin{tabular}{|c|c|c|c|c|c|c|c|c|c|c|}
\hline \multirow{2}{*}{ Femur } & \multicolumn{5}{|c|}{ Males } & \multicolumn{5}{|c|}{ Females } \\
\hline & $\mathbf{N}$ & $\mu(\mathrm{cm})$ & SD $(\mathrm{cm})$ & Max. (cm) & Min. (cm) & $\mathbf{N}$ & $\mu(\mathrm{cm})$ & $\mathrm{SD}(\mathrm{cm})$ & Max. (cm) & Min. (cm) \\
\hline MLF & 238 & 43.638 & 4.168 & 50.5 & 38.4 & 205 & 40.253 & 2.045 & 45.2 & 35.0 \\
\hline GTH & 227 & 9.783 & 0.812 & 11.3 & 8.4 & 190 & 8.775 & 0.516 & 10.3 & 7.9 \\
\hline GLT & 225 & 6.722 & 0.747 & 9.8 & 5.2 & 187 & 5.988 & 0.530 & 7.6 & 4.8 \\
\hline DLM & 233 & 8.200 & 0.853 & 10.3 & 6.0 & 200 & 7.406 & 0.469 & 8.8 & 5.1 \\
\hline
\end{tabular}

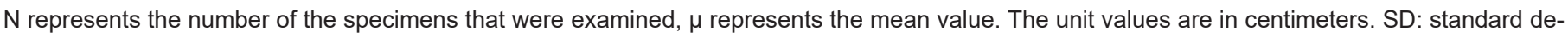

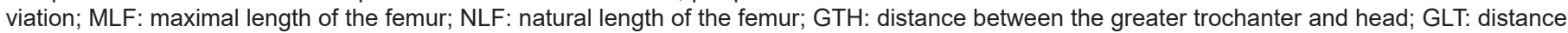

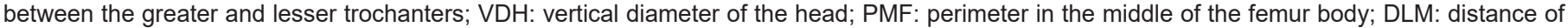
lateral and medial between the outermost extremities of the two condyles of the femur.

predictor or independent variable is interval based in nature. All analyses were completed using the $\mathrm{R}$ software environment (R Core Team 2019-v.R-3.4.2) for statistical computing and graphics [1].

\section{Results}

The measurements of all the dimensions were accomplished using an osteometric board (for distances between two points), sliding calliper (for maximum diameters) and metal tape for the circumferences. Regarding the MLF, this was defined as the distance between the outermost superior point on the femur's head and the most inferior mark resting on the distal condyles, using the osteometric board.

From the data (Tables 1 and 2), it can be deduced that all the mean values, diameters and indices of the males were greater compared with those of the females. The analysis also revealed that the standard deviation of the diameters and indices cascade the values of the diameters for both sexes.

Because we relied on the statistical parameters' mean values and standard deviations of the two samples, we used discriminant analysis to provide more accurate and reliable results. The more specific mean values and standard deviations of the samples correspond to those of the general population, which is unknown; moreover, we assume they follow the normal distribution.

According to the diagram (Fig. 1), the two curves correspond to the normal distribution of males (blue) and females (red) according to the mean value and standard deviation of
Table 1 for the MLF; the value that separates the two sexes is 41.37 , representing the decision point. This means that, if we have a femur that is of unknown sex and the MLF is higher than 41.37 , it most probably belongs to a male. This is because the common error for this variable is $29.29 \%$ (Table 3 ), which is quite high. The error is calculated as the area of the red and blue surface under the normal curves. Thus, if the length of MLF is less than 41.37, we conclude that belongs to a woman with error $29.29 \%$ (area of the red shape) which corresponds to the probability of the event: "the length of MLF that belongs to a woman is higher than 41.37 '. On the contrary, if the length of MLF is higher than 41.37 , we conclude that belongs to a man with error $29.29 \%$ (area of the blue shape) which corresponds to the probability of the event: "the length of MLF that belongs to a man is less than 41.37 ". The cut-off point was selected in order to be equal with the two errors mentioned above.

According to Table 3, we can conclude that the probability of error is quite high in all cases except the VDH value, which has an acceptable percentage of error of $14.39 \%$ (Fig. 2) and can be used as a safe criterion. In contrast, the IRFs have an error of $46.02 \%$ (Fig. 3), so these values do not represent a reliable measurement for sex identification.

\section{Discussion}

The skeletal remains of the lower extremities can assist in gender identification and be crucial in forensic anthropology; moreover, the femur is considered the most important bone from an anthropometrics perspective. In the erect position, it

Table 2. The Mean Values and the Standard Deviation of the Bone Robustness

\begin{tabular}{|c|c|c|c|c|c|c|c|c|c|c|}
\hline \multirow{2}{*}{ Index } & \multicolumn{5}{|c|}{ Males } & \multicolumn{5}{|c|}{ Females } \\
\hline & $\mathbf{N}$ & $\mu(\mathrm{cm})$ & $\mathrm{SD}(\mathrm{cm})$ & Max. (cm) & Min. (cm) & $\mathbf{N}$ & $\mu(\mathrm{cm})$ & SD (cm) & Max. (cm) & Min. (cm) \\
\hline IRF 1 & 181 & 21.559 & 1.627 & 31.8 & 14.1 & 160 & 21.246 & 1.509 & 24.8 & 18.5 \\
\hline
\end{tabular}

$\mathrm{N}$ represents the number of the specimens that were examined. $\mu$ represents the mean value. SD: standard deviation; IRF 1: index of robustness of the femur 1; IRF 2: index of robustness of the femur 2. 


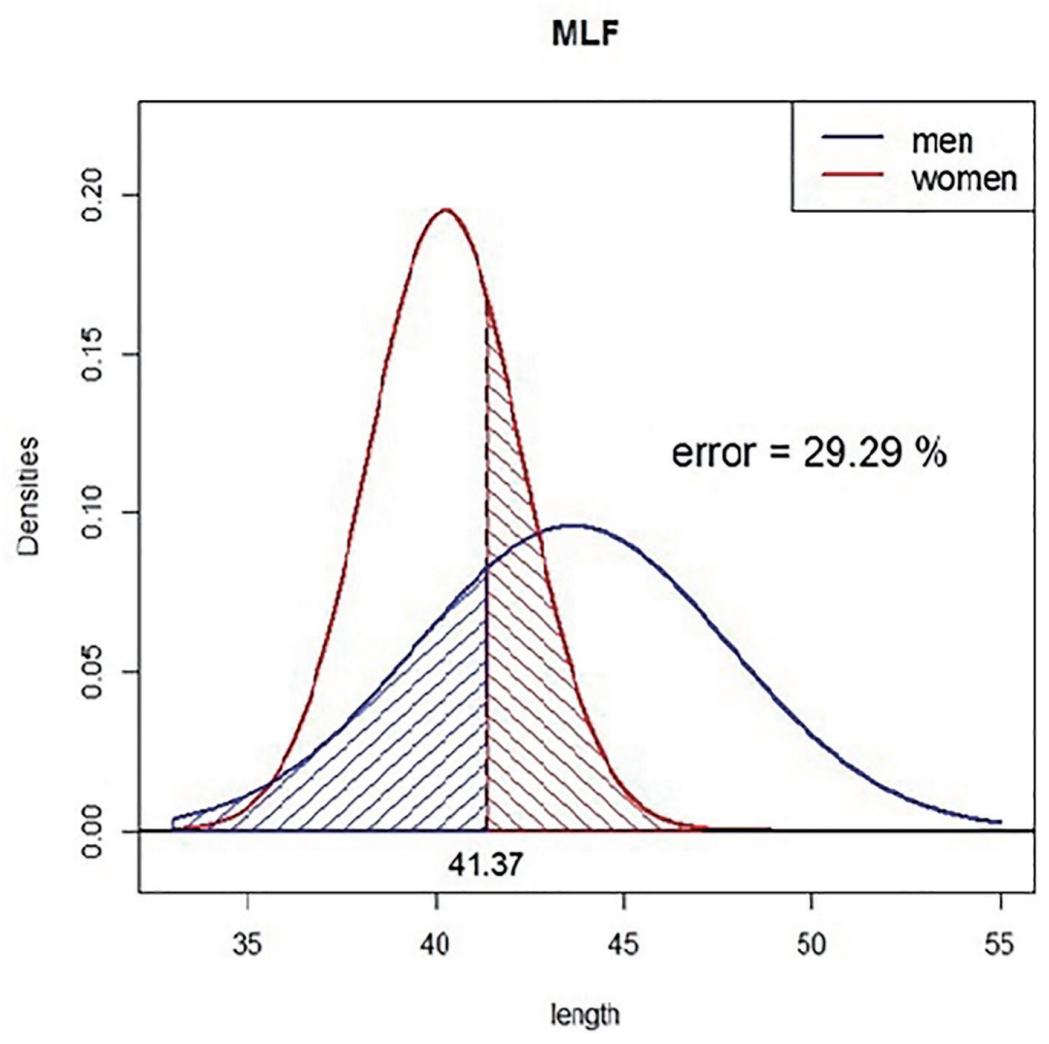

Figure 1. The normal distribution curves for the maximal length of the femur (MLF). The blue represents the male and the red represents the female. Decision point is 41.37 and the error is $29.29 \%$ for sex estimation.

has a downward oblique direction with a slight posterior position, converging with its reversal bone. This angled course is more substantial in females due to the bigger amplitude of the pelvic floor [2]. In general, adult male bones are more ragged and compact than female bones are, with the latter preserving a more childlike appearance; however, these differences tend

Table 3. The Point of Decision and the Error of the Measurements When Comparing Between the Two Sexes Using the Discriminant Analysis

\begin{tabular}{lll}
\hline & Point of decision $(\mathbf{c m})$ & Error \\
\hline MLF & 41.37 & $29.29 \%$ \\
NLF & 39.94 & $29.60 \%$ \\
GTH & 9.17 & $22.39 \%$ \\
GLT & 6.29 & $28.27 \%$ \\
VDH & 4.46 & $14.39 \%$ \\
PMF & 8.56 & $32.81 \%$ \\
DLM & 7.69 & $27.41 \%$ \\
\hline
\end{tabular}

MLF: maximal length of the femur; NLF: natural length of the femur; GTH: distance between the greater trochanter and head; GLT: distance between the greater and lesser trochanters; VDH: vertical diameter of the head; PMF: perimeter in the middle of the femur body; DLM: distance of lateral and medial between the outermost extremities of the two condyles of the femur. to be eradicated due to age-related decay [3].

Our measurements can also be applied to fragmented remains if at least the radius head diameter is intact and can be measured. At least one study has shown that even cremated remains can demonstrate a high degree of accuracy in sex determination. Similarly, the measurement of the femur perimeter assists in sex identification in inadequately preserved skeletons and bones belonging to more than one person $[4,5]$.

Clinically, fractures of the femur tend to have an epidemic character in Western societies. More specifically, one in three females and one in six males have suffer at least one such fracture up to their $90 \mathrm{~s}$, while $25 \%$ of the hospitalizations in orthopedic departments are due to these fractures. In the past 50 years, there have been breakthrough discoveries in techniques for total knee and hip replacement, operations in which it is crucial to be familiar with the anatomical differences of femurs between genders [6-9]. Thus, there must be extensive data collection from all races and ages to have a more accurate design of all types of prostheses of the proximal and distal femur.

Another issue regarding femur diameters is ethnic variation, which is well documented not only between different races but also among countries with varied populations [10]. More specifically, our study revealed that the MLF parameter of the northern Greek population is the shortest in Europe, but it is longer than the values found in Korean, Japanese and Taiwanese people; furthermore, it is comparable to the south Indian and much shorter than the African American populations 


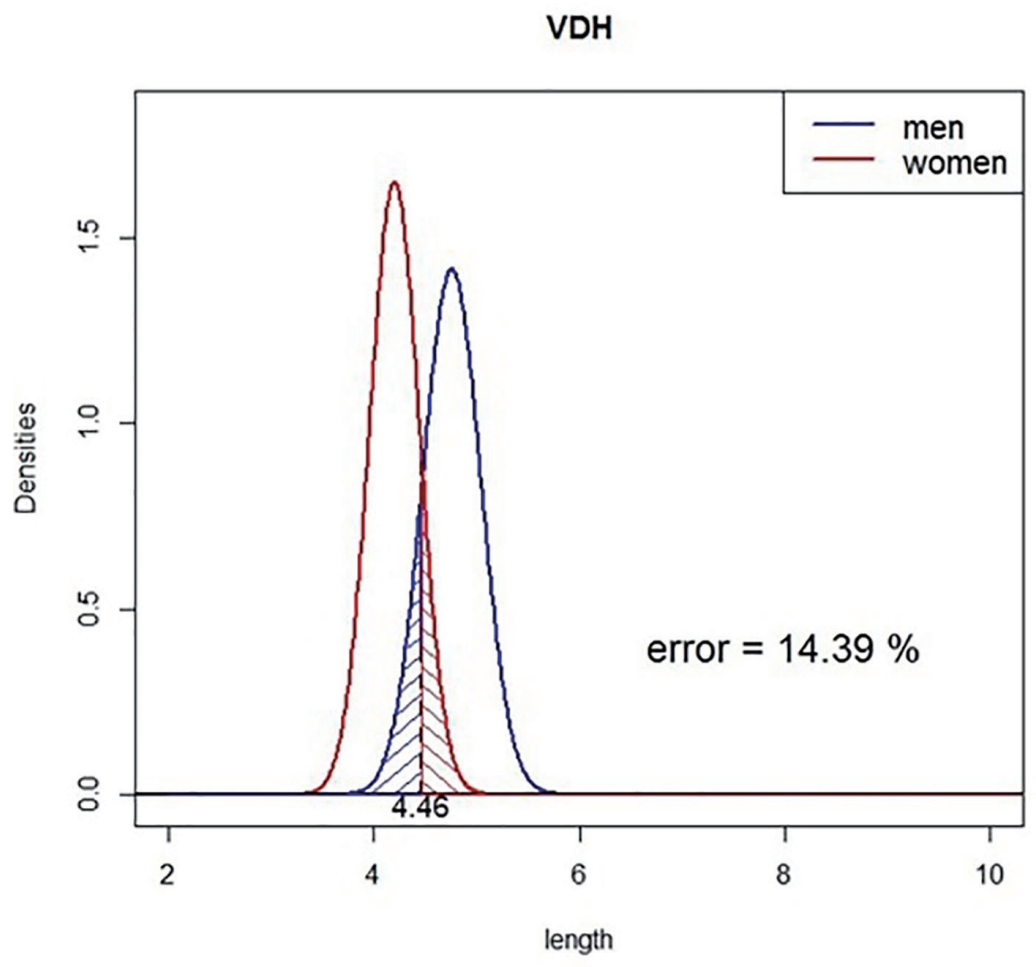

Figure 2. The normal distribution curves for the vertical diameter of the head (VDH). The blue represents the male and the red represents the female. Decision point is 4.46 and the error is $14.39 \%$ for sex estimation.

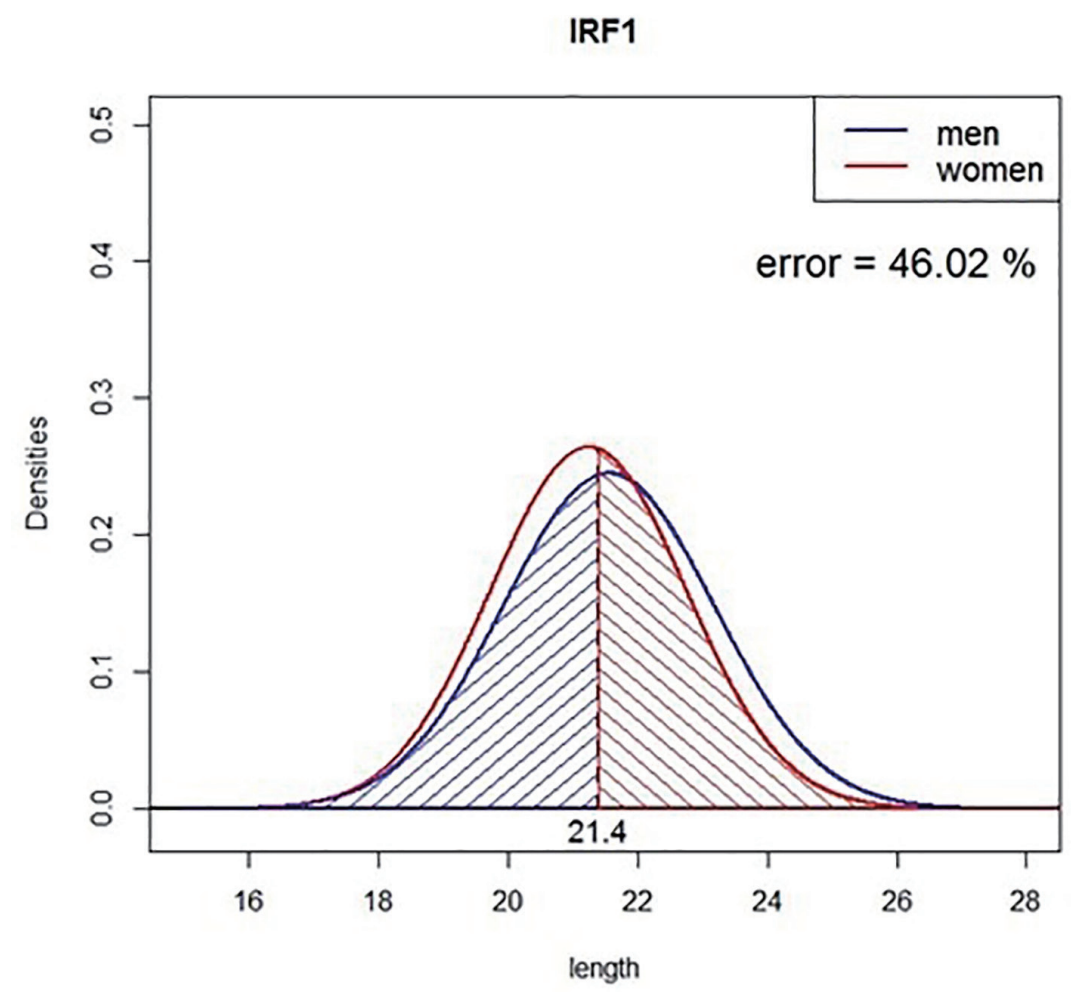

Figure 3. The normal distribution curves for the index of robustness (IRF). The blue represents the male and the red represents the female. Decision point is 21.4 and the error is $46.02 \%$ for sex estimation. 
[11-13].

As with all studies, there are strengths and limitations in our analysis. One strength is the large number of skeletal remains used for the study, which strengthened the statistical power, while the use of discriminant analysis gave us the opportunity to obtain more reliable results. Regarding the limitations, we relied on the mean value and standard deviation due to limited access to the full data of the measurements.

Finally, in our study, the mean values of all the surveying parameters, such as MLF, were greater in males than they were in females, revealing that women have much smaller bone dimensions than their male analogues do. Thus, it is crucial for forensic medicine to determine the anatomical standards of each population to assign a gender to an unidentified femur as much as possible using three parameters conducive to $80 \%$ certainty [14-18]. Our research revealed that one parameter is to be trusted, namely, the VDH, which gives a much more precise determination of sex.

\section{Acknowledgments}

The authors wish to express their gratitude to the ossuary of Thessaloniki for granting access to their human skeleton collection.

\section{Financial Disclosure}

This research received no grant funding from any funding agency in the public, commercial or not-for-profit sectors.

\section{Conflict of Interest}

The authors have no conflict of interest to report.

\section{Informed Consent}

Not applicable.

\section{Author Contributions}

George Karavasilis contributed to the statistical analysis. All authors contributed to the conception and design of the manuscript as well as revising it critically for important intellectual content and final approval of the version.

\section{References}

1. R Core Team [online]. R: A language and environment for statistical computing. R Foundation for Statistical Computing, Vienna, Austria. Available from: htts://www.Rproject.org/.
2. Tague RG. Variation in pelvic size between males and females. Am J Phys Anthropol. 1989;80(1):59-71.

3. Ruff CB, Hayes WC. Sex differences in age-related remodeling of the femur and tibia. J Orthop Res. 1988;6(6):886896.

4. Cavazzuti C, Bresadola B, d'Innocenzo C, Interlando S, Sperduti A. Towards a new osteometric method for sexing ancient cremated human remains. Analysis of Late Bronze Age and Iron Age samples from Italy with gendered grave goods. PLoS One. 2019;14(1):e0209423.

5. Kanchan T, Krishan K. Anthropometry of hand in sex determination of dismembered remains - A review of literature. J Forensic Leg Med. 2011;18(1):14-17.

6. Gillespie RJ, Levine A, Fitzgerald SJ, Kolaczko J, DeMaio M, Marcus RE, Cooperman DR. Gender differences in the anatomy of the distal femur. J Bone Joint Surg Br. 2011;93(3):357-363.

7. Dattani R. Femoral osteolysis following total hip replacement. Postgrad Med J. 2007;83(979):312-316.

8. Unnanuntana A, Toogood P, Hart D, Cooperman D, Grant RE. Evaluation of proximal femoral geometry using digital photographs. J Orthop Res. 2010;28(11):1399-1404.

9. Verma M, Joshi S, Tuli A, Raheja S, Jain P, Srivastava P. Morphometry of Proximal Femur in Indian Population. J Clin Diagn Res. 2017;11(2):AC01-AC04.

10. Siddiqi N. Comparison of osteometric femoral bone dimensions among the South Africans of different ethnic groups and South African whites. Egypt J Forensic Sci. 2013; 3:8-14.

11. Cho HJ, Kwak DS, Kim IB. Morphometric Evaluation of Korean Femurs by Geometric Computation: Comparisons of the Sex and the Population. Biomed Res Int. 2015;2015:730538.

12. Solan S, Kulkarni R. Estimation of total length of femur from its fragments in South Indian population. J Clin Diagn Res. 2013;7(10):2111-2115.

13. Jayachandra P, Lakshmi D, Sobha D. Osteometric studies on human femurs. IOSR J Dent Med Sci. 2014;13:34-39.

14. Kwak DS, Han S, Han CW, Han SH. Resected femoral anthropometry for design of the femoral component of the total knee prosthesis in a Korean population. Anat Cell Biol. 2010;43(3):252-259.

15. Patil G, Kolagi S, Ramadurg U. Sexual dimorphism in the humerus: south Indians. J Clin Diagn Res. 2011;5:534538.

16. Basic Z, Anteric I, Vilovic K, Petaros A, Bosnar A, Madzar T, Polasek O, et al. Sex determination in skeletal remains from the medieval Eastern Adriatic coast discriminant function analysis of humeri. Croat Med J. 2013;54(3):272-278.

17. Kranioti EF, Michalodimitrakis M. Sexual dimorphism of the humerus in contemporary Cretans - a populationspecific study and a review of the literature*. J Forensic Sci. 2009;54(5):996-1000.

18. Rios Frutos L. Metric determination of sex from the humerus in a Guatemalan forensic sample. Forensic Sci Int. 2005;147(2-3):153-157. 